\title{
Dimensiones de integración social en población colombiana y cubana que vive en Quito, Ecuador*
}

\section{Dimensions of Social Integration in the Colombian and Cuban Population Living in Quito, Ecuador}

Recepción: 28 Agosto 2017 | Aprobación: 06 Noviembre 2017

\author{
Carlos Reyes Valenzuela ${ }^{\mathrm{a}}$ \\ Universidad Politécnica Salesiana, Ecuador \\ ORCID: http://orcid.org/0000-0001-8911-700X \\ Francisco Jaramillo Tejada \\ Pontificia Universidad Católica del Ecuador, Ecuador \\ LiLIANA Jayo Suquillo \\ Pontificia Universidad Católica del Ecuador, Ecuador \\ Marie-France Merlyn Sacoto \\ Pontificia Universidad Católica del Ecuador, Ecuador \\ María José Martos-Méndez \\ Universidad de Málaga, España
}

a Autor de correspondencia. Correo electrónico: creyes@ups.edu.ec

Para citar este artículo: Reyes Valenzuela, C., Jaramillo Tejada, F., Jayo Suquillo, L., Merlyn, M. -F., \& Martos, M. J. (2017). Dimensiones de integración social en población colombiana y cubana que vive en Quito, Ecuador. Universitas Psychologica, 16(5), 1-14. https:// doi.org/10.11144/Javeriana.upsy16-5.disp

\section{RESUMEN}

La integración social (IS) se presenta como un fenómeno clave en la complejidad de las sociedades actuales. Son escasas las investigaciones que consideran una perspectiva psicosocial para evaluar la IS. En el presente trabajo, se plantea una propuesta que tiene en cuenta tales dimensiones en población colombiana con desplazamiento forzado y cubana con migración económica, residentes en Quito (Ecuador). Participaron 255 personas a quienes se evalúan la anomia y arraigo social. Se encuentra que, en ambos grupos de participantes, la presencia o ausencia de trabajo condiciona la anomia o arraigo social. Estos resultados presentan implicaciones sobre diversas políticas de inmigración, en las cuales la integración social requeriría incorporar dimensiones psicosociales para abordar tal proceso.

Palabras clave

integración social; migración forzada y económica en Ecuador; políticas de integración.

\begin{abstract}
Social Integration (SI) is presented as a key phenomenon in the complexity of today's societies. There are few investigations that considered psychosocial perspective to evaluate SI and here we propose a proposal that considers such dimensions in Colombian population with forced displacement and Cuban with economic migration residing in Quito, Ecuador. 255 persons participated who are evaluated anomia and social roots. The models of mediation show that in both groups of participants, the presence or absence of work conditions the social anomia or rootedness. These results have implications for various immigration policies, in which social integration requires incorporating psychosocial
\end{abstract}


dimensions to address such a process. Keyword social integration; forced and economic migration in Ecuador; integration policies Introducción

Keywords

social integration; forced and economic migration in Ecuador; integration policies.

\section{Introducción}

La noción de integración social (IS) representa un complejo proceso social que pone de manifiesto las relaciones y vínculos entre grupos sociales. Aunque históricamente su origen puede relacionarse con la ideología que origina la Revolución Francesa en relación con la equidad, igualdad y fraternidad entre ciudadanos, fue Emile Durkheim uno de los primeros en abordarla en relación con el desarrollo de los Estados modernos. Durkheim instala la discusión en torno al modo cómo las sociedades modernas producen y mantienen el orden social. La IS, siguiendo al autor, haría referencia a los medios y modos de organización y regulación de la sociedad, a través de normas que promueven el orden social (Durkheim, 1989). La carencia de estas normas, por tanto, pone en evidencia la desintegración social de una sociedad.

Esta inicial concepción de IS posibilita un creciente marco conceptual desde la sociología, que la sintetiza como un tránsito de los individuos para incorporarse a una estructura social y como un fin, en la medida en que reporta un cierto orden social (Cohen, Gottlieb, \& Underwood, 2000; House, Umberson, \& Landis, 1988). De allí que el proceso de ruptura o debilitamiento de los vínculos sociales, esto es, la exclusión social de los individuos, pone en evidencia una estructura de desintegración social (Castel, 1997; Laparra et al., 2007).

\section{Una perspectiva psicosocial de la integración social}

Coincidiendo con un enfoque sociológico, la perspectiva psicosocial de la IS concibe la relación entre lo individual y lo social desde una dinámica de mutua constitución (Asún, Alfaro, \& Morales, 1994). No obstante, lo distintivo en esta concepción es la importancia que se atribuye a los procesos de percepción subjetiva en la dinámica de relación con objetos sociales, de modo que esta interacción no es mecánica ni está predeterminada únicamente por aspectos socioeconómicos (Asún et al., 1994; Asún et al., 1998). Así, la IS es entendida no solo en una dimensión de oportunidad y acceso a la estructura económico-social (plano objetivo), sino también considera el intercambio que realiza el individuo con su entorno simbólico y cultural (plano subjetivo). Conjuntamente, la noción de IS se constituye en la relación que se establece en dichos planos, en cuanto a las dimensiones macro y microsociales, en un contexto histórico-cultural. De esta manera, una definición teórica-metodológica aborda la IS como "una posición, estado o forma de relación entre sujetos colectivos o individuales y el conjunto social, que ocurre en distintos planos de la organización social, económica y cultural" (Asún et al., 1998, p. 58). Esta definición posibilita reconocer dos ámbitos de la IS: uno subjetivo-objetivo y otro micro y macrosocial.

La IS se puede observar en su dimensión objetiva a través del grado de participación activa de un individuo en los medios sociales donde transcurre su desarrollo, es decir, el nivel en el cual el individuo hace uso de los medios sociales que le permitirían cumplir ciertos fines. La dimensión objetiva/microsocial plantea describir cómo los individuos perciben su red social y si esta les provee de apoyo, en tanto la dimensión objetiva/macrosocial explora cómo las personas participan en escenarios sociales. De este modo, un plano objetivo se evalúa a través de la estructura de red social y la participación social, respectivamente. En tanto, la IS desde una dimensión subjetiva se comprende en relación con la percepción individual del medio sociocultural, esto es, al valor o interpretación que el individuo le asigne al contexto social donde se posiciona. Así, se relaciona con las actitudes, motivaciones, pautas de comportamiento, proyectos de vida y relaciones emocionales que los individuos van construyendo dentro de la red de relaciones 
sociales en la cual se inscribe. De allí que una dimensión subjetiva/microsocial identifica el apoyo social percibido y una dimensión subjetiva/macrosocial se aborda por medio de la noción de anomia subjetiva (Asún et al., 1998). Esta operacionalización evalúa inicialmente la IS en jóvenes, tanto respecto al consumo de drogas (Asún et al., 1998) como en conductas delictivas (Valdenegro, 2005). Los anteriores trabajos se enfocaron fundamentalmente en valorar la dimensión subjetiva de la IS, a partir del interés en evaluar a jóvenes con y sin comportamientos de riesgo y de la dificultad de configurar instrumentos de medición en la variable de redes sociales. Para el estudio aquí propuesto del fenómeno migratorio, esta línea es la que se mantiene en el presente trabajo.

\section{Estudios sobre integración social en población migrante}

Hay numerosas investigaciones que abordan la integración social en inmigrantes, desarrolladas principalmente en España, entre otras, con población latinoamericana. Herrero, Gracia, Fuente y Lila (2012) consideran que la integración social se relaciona con el bienestar psicosocial de los inmigrantes, convirtiéndose en un sostén importante para estas personas, especialmente, los recién llegados a la comunidad. Asimismo, la integración social puede indicar un nivel de adaptación y participación en la sociedad receptora, ya que se ha observado que el aislamiento social y poco contacto con la comunidad autóctona puede incidir negativamente en la salud y el bienestar de los migrantes. Sus niveles de estrés pueden aumentar ya que no cuentan con apoyo social para poder resolver situaciones vitales estresantes (Cohen et al., 2000). Numerosos estudios muestran que los inmigrantes son un grupo particularmente vulnerable al estrés debido al proceso de adaptación a la sociedad receptora (Cuellar, Bastida, \& Braccio, 2004; Zarza \& Sobrino, 2007), y la participación social tiene para ellos un significado importante (Herrero \& Gracia, 2007) al incrementar su satisfacción con la vida y contribuir de forma relevante a su proceso de integración en la sociedad de acogida.

Así, las personas migrantes pueden aumentar su bienestar psicológico por medio de apoyo social de personas cercanas (pareja, familia y amigos íntimos), aunque su integración en un contexto social más amplio es también importante para su satisfacción y bienestar general (Cowen, 2000; Herrero et al., 2012). Por otro lado, algunas influencias contextuales, por ejemplo, las características de los barrios donde residen los inmigrantes, constituyen un importante factor que puede influir de forma determinante en los niveles de integración social (Gracia, García, \& Musitu, 1995; Gracia \& Herrero, 2004). Por tanto, entender aquellos factores que pueden promover o dificultar su nivel de integración se convierte en un área fundamental de investigación (Herrero et al., 2012).

\section{Situación de la migración en Ecuador para población colombiana y cubana}

Ecuador se ha instituido históricamente como un país receptor de migración, especialmente desde la segunda parte del s. XX. Esta dinámica se vio abruptamente modificada a finales de la década de los 90 , que lleva paralelamente tanto a un masivo éxodo de nacionales ecuatorianos principalmente con destino a EE. UU., España e Italia- como a la llegada de migrantes de países fronterizos, predominantemente provenientes de Colombia. Hasta el año 2006, la gran mayoría de migración colombiana se relacionaba como resultado del conflicto armado, de la cual una minoría vivía en condición de refugiada reconocida por el Estado ecuatoriano. Con la llegada al Gobierno de Rafael Correa Delgado, a partir de 2007, se implementó una política de recepción y reconocimiento de la población desplazada y migrantes en general, con profundas reformas en planes y normativas -incluyendo la Constitución en el año 2008- al reconocer la libre movilidad, ciudadanía universal e igualdad de derechos. Estos cambios han posicionado a Ecuador como el país que proporciona mayor 
refugio en Latinoamérica, del cual un $98 \%$ de este contingente proviene de Colombia (Alto Comisionado de las Naciones Unidas para los Refugiados [ACNUR], 2014). De acuerdo a cifras del ACNUR (2016), 57312 personas colombianas son refugiadas reconocidas, 233 049 son solicitantes de refugio y alrededor de 500 personas mensuales son solicitantes, de las cuales es levemente superior la población femenina (Herrera, 2008). No obstante, en los últimos años, la situación de recepción para población desplazada colombiana ha presentado abruptos cambios que en la actualidad se inscriben en un entorno de doble exclusión para personas desplazadas. Primero, porque se identifica una dinámica de un contexto expulsor en Colombia (Jaramillo, 2008) y, posteriormente, de una recepción progresivamente excluyente en Ecuador. El detonante de la huida lo sigue configurando el estado de violencia generalizada, caracterizada por amenazas, asesinatos selectivos, reclutamiento forzado y hostigamiento económico desde actores armados, que obliga a las personas a abandonar su lugar de origen. Por el contrario de lo que podría esperarse, estos aspectos que siguen ocurriendo aún luego de la firma en 2016 del Acuerdo Final para la Terminación del Conflicto y la Construcción de una Paz Estable y Duradera suscrito entre el Gobierno de la República de Colombia y las Fuerzas Armadas Revolucionarias de Colombia (las FARC). Conjuntamente, la permanencia de población desplazada colombiana se ha visto alterada por las condiciones políticas y económicas actuales de Ecuador, que está presentando una paulatina fragilidad en su economía, basada en el dólar, que lo hace más adverso para la subsistencia y acceso al trabajo para estas personas (Sozanski, Sarmiento, \& Reyes, 2016). Además, con la firma del Acuerdo anteriormente señalado, hay inseguridad en la población desplazada por una disminución del apoyo que ACNUR ha dedicado y, del mismo modo, se ha observado una baja progresiva de aceptación a solicitudes de refugio.

En relación con la población cubana que vive en Ecuador, la gran mayoría se encuentra por "motivos económicos", migración que se ha producido durante la última década, justamente avalada por la normativa constitucional de Ecuador (Correa, 2013). De acuerdo a cifras del Ministerio de Relaciones Exteriores y Movilidad Humana (2016) entre el 2012 y 2016 se han otorgado 26936 visas para residentes temporales, 16738 visados para residentes permanentes y 697 personas han adoptado la nacionalidad ecuatoriana. De acuerdo con Arcentales (2012), la población migrante cubana se concentra en Quito, es mayoritariamente masculina, con una edad comprendida entre los 20 y 49 años, caracterizada por presentar cualificación en sus estudios. No obstante estas características, hay una percepción de sobrevaloración en el número, lo que ha promovido una sensación de alarma en la sociedad receptora respecto a la migración cubana (Correa, 2013). A esto se añade que la experiencia de migración cubana en Ecuador presenta una permanente vulnerabilidad, ya sea laboral o por la regularización de su condición migratoria. Precisamente, a pesar del ordenamiento jurídico vigente, se han llevado a cabo procesos de exclusión y deportación, uno de los cuales se produjo masivamente en julio de 2016. Como señala Correa, "el contexto (ecuatoriano) promueve una realidad traumática que no solo retarda cualquier proceso de integración social, sino que favorece la reproducción de lógicas de guetorización y de rechazo invertido de los colectivos migrantes frente a sociedades huésped" (2013, p. 45).

En este contexto migratorio, Ecuador ha emitido una Ley Orgánica de Movilidad Humana (Asamblea Nacional del Ecuador, 2017) que sigue reafirmando los principios constitucionales de reconocimiento de derechos para la población migrante, el derecho a migrar, los derechos de asilo y refugio y la no devolución de personas. También, reconoce los principios de ciudadanía universal, libre movilidad humana, prohibición de criminalización, igualdad ante la ley y no discriminación, que promueve derechos y obligaciones a todo extranjero. No obstante, han surgido críticas respecto al real reconocimiento de la igualdad, ya que se reconoce únicamente a extranjeros con visados, delimita la definición de sudamericanos a países integrantes del UNASUR 
y, de este modo, jerarquiza una categoría de sudamericanos y otra de extranjeros (Arcentales, 2017). Por lo tanto, se requiere una condición migratoria regular para acceder a los derechos establecidos en la Ley Orgánica de Movilidad Humana, orientación permanente en muchos países pero que bajo la Constitución ecuatoriana equivale a una violación a los principios de igualdad y no discriminación (Arcentales \& Chávez, en prensa).

Atendiendo a estas consideraciones, se plantea como pertinente y relevante que las investigaciones en movilidad social indaguen sobre los mecanismos que explican las dimensiones de la integración social, examinando variables micro y macrosociales que inciden en el proceso migratorio. En el presente estudio, se plantea el objetivo de explorar las condiciones que promueven la anomia y el arraigo social de población colombiana y cubana que reside en Quito e investigar qué factores están interviniendo en su presencia o ausencia. Se presenta una adaptación de los trabajos que se han realizado con población migrante y se adopta la operacionalización de IS desde una perspectiva psicosocial, la cual se orientará únicamente al plano subjetivo y su relación con las dimensiones micro y macrosocial. El vínculo con la sociedad de acogida para población migrante puede identificarse a partir de una dinámica conflictiva y/o de satisfacción que condiciona su integración. Así, desde un plano de una dinámica de dificultades, se considera la IS en población migrante en un plano subjetivo/microsocial como los problemas percibidos con la sociedad de acogida y en un plano subjetivo/macrosocial como anomia subjetiva. Por otro lado, desde una perspectiva de satisfacción, la IS en migrantes en un plano subjetivo/microsocial corresponde a un vínculo satisfactorio percibido con la sociedad de acogida y, en un plano subjetivo/macrosocial, al arraigo social. Estas relaciones estarán mediadas por características y costumbres de la población migrante y por factores contextuales propios de la sociedad de acogida. De esta manera, se plantean dos objetivos para cada grupo de migrantes, esto es, la población colombiana y cubana: explorar las condiciones que describen una relación problemática de estos grupos con la sociedad de acogida y anomia subjetiva (objetivo 1) y las variables que median la relación entre satisfacción y arraigo social (objetivo 2). Se espera explorar la influencia que las características específicas de la población migrante, tales como origen de la migración, las costumbres y estilo de vida, tienen sobre una dinámica problemática o satisfactoria con la sociedad de acogida en Quito. Por último, se evalúa la influencia de factores contextuales tales como el acceso o no a una actividad laboral remunerada.

\section{Método}

\section{Participantes}

El estudio está conformado de 253 participantes, 139 hombres (54.9\%) y 114 mujeres (45.1\%). Sus edades varían entre 18 y 75 años $(M=33.67$ y $D E=8.99)$. Los participantes colombianos $(N=105)$, provienen de los departamentos de Cundinamarca (Bogotá), Nariño, Valle del Cauca y Antioquia, entre otros. El principal motivo de salida de Colombia es la situación de violencia que los ha llevado a la solicitud de refugio en el Ecuador, razón por la que son atendidos en la institución Asylum Access Ecuador (AAE). Por su parte, los participantes cubanos $(N=148)$ provienen de La Habana, Santiago y Pinar del Río, entre otras ciudades. El principal motivo de salida del país es económico (89 \%) y el restante $11 \%$ aduce como argumento la situación política cubana. En la Tabla 1 se pueden observar las principales características de la muestra.

\section{Instrumentos}

\section{Datos sociodemográficos}

Se indaga por aspectos tales como sexo, edad, lugar de procedencia, estado civil y nivel educativo. 


\section{Condición migratoria}

A los participantes colombianos se les consultó de acuerdo a la solicitud de refugio que realizan en Ecuador. Las opciones de respuesta posibles eran cinco: refugio reconocido, solicitud, negación, indocumentado y otra visa. Entre los participantes cubanos, las opciones de respuestas eran dos: situación legal regularizada y no regularizada.

\section{Tiempo de residencia en Ecuador}

Se planteó la siguiente pregunta: "¿Cuál es el año de llegada a Ecuador?”. Esto posibilitó agrupar las respuestas en tres categorías: $1=$ menos de tres años, $2=$ entre 3 y 6 años y $3=$ más de 7 años.

\section{Situación laboral}

Estaba conformada por tres opciones de respuesta: trabajo por cuenta propia, por cuenta ajena y desempleado.

\section{Mantención de costumbres culturales}

Se crearon tres reactivos que evaluaron tres aspectos de costumbres culturales: tradiciones familiares, comidas y celebraciones del país de origen. Se diseñaron los siguientes reactivos: "iConserva en Quito las costumbres asociadas a tradiciones familiares?", “iConserva en Quito las costumbres de comidas de su país de origen?" y "iMantiene en Quito las costumbres de las celebraciones de su país de origen?" La consistencia interna fue satisfactoria $(\alpha=0.83)$. Las opciones de respuesta se califican con 0 (No) y $1(\mathrm{~S} i)$.

Adaptación al estilo de vida local ([SCAS], Ward Ë Kennedy, 1999)

Adaptada al castellano por De Luca, Bobowick y Basabe (2007). Se evaluaron cinco ítems del factor de Aprendizaje Cultural y Comunicación, en el que se consultaron los siguientes reactivos: "Acostumbrarse a la comida local", "Entender las bromas y el sentido del humor de las personas autóctonas", "Entender el acento local, la lengua, el idioma", "Comprender el punto de vista de la cultura local" y "Acostumbrarse al ritmo de vida". Se obtiene una consistencia interna satisfactoria $(\alpha=0.84)$. Las opciones de respuesta varían entre 1 (ninguna dificultad) a 5 (extrema dificultad).

Problemas percibidos en vínculo con sociedad de acogida

Se diseñaron cuatro ítems que evaluaban problemas con personas de Quito. Para ello se preguntaron los siguientes reactivos: "iCree que los ecuatorianos rechazan a los extranjeros?", "iHa tenido problemas en Quito por el hecho de ser extranjero/a?", "iDónde ha ocurrido ese problema?", "iLe han negado alguna vez el arriendo de una vivienda por ser extranjero/a?". La consistencia interna fue aceptable $(\alpha=0.71)$. Las opciones de respuesta se califican con $0(\mathrm{No})$ y 1 (Sí), a excepción en ítems donde se pedía una especificación del problema.

Vínculo satisfactorio percibido con la sociedad de acogida

Se diseñaron tres ítems que evalúan el vínculo satisfactorio percibido que el participante presenta con población ecuatoriana que vive en Quito. Se confeccionaron estos reactivos: "iPresenta amigos entre ecuatorianos?", "iHa contado con ayuda de ecuatorianos cuando tiene problemas? ¿Ha visitado amigos ecuatorianos en sus hogares?" Se halló una buena consistencia interna $(\alpha=0.83)$. Las opciones de respuesta corresponden a $0\left(\mathrm{No}_{0}\right)$ y $1(\mathrm{~S} i)^{2}$.

Escala de Anomia Subjetiva (Asún et al., 1998)

Se evaluó la integración social a través de la anomia subjetiva, con 20 ítems. La escala presenta cuatro subdimensiones: extrañamiento, exclusión social, incertidumbre y degradación. 
Se siguió el procedimiento de recodificación, señalado en un estudio con jóvenes (Aceituno et al., 2009), donde una alta puntuación se asocia a mayor anomia. Se encontró una consistencia interna aceptable $(\alpha=0.75)$. Las alternativas de respuesta varían así: 1 (de acuerdo), 2 (no sé) y 3 (en desacuerdo).

Escala de Arraigo Social (Torrente, RuizHernández, Ramírez, Eु Rodríguez, 2011)

Se midió el arraigo social que se utiliza en población inmigrante, en tres dimensiones: arraigo cultural, ecológico y social/laboral. Se mantuvo la consigna de los autores: "A continuación se le presenta una serie de enunciados relacionados con su vida en Quito (Ecuador). Por favor, indique hasta qué punto se siente vinculado o siente como suyo cada uno de ellos". La escala presenta una consistencia interna excelente $(\alpha=0.91)$. Las opciones de respuestas varían entre 1 (Nada en absoluto) a 5 (Mucho).

\section{Procedimiento}

Se realizó inicialmente una prueba piloto con población migrante en Quito para evaluar la pertinencia y comprensión de los ítems. Se efectuaron pequeños cambios al instrumento original, aunque se observó la necesidad de aclarar el objetivo y fuente del estudio, dada cierta perspicacia en algunos participantes. En una segunda etapa, se realizó una puesta de contacto con instituciones que trabajaran con población migrante en Quito. Con población colombiana, se establecieron reuniones con Asylum Access Ecuador (AAE), entidad que estableció una normativa para aplicar las encuestas dentro de sus actividades. Fueron capacitados cinco encuestadores, quienes además recibieron capacitación del equipo de AAE. En este caso, el periodo de aplicación se realizó entre los meses de mayo a julio de 2014.

En relación con la población cubana, el contacto se estableció a través de un informante clave que facilitó el acercamiento inicial, específicamente, en un barrio de Quito. Los referidos encuestadores establecieron contacto personal con la población cubana, lo que facilitó la aplicación de la encuesta. El procedimiento incluyó la descripción de los objetivos del estudio, la lectura y firma de un consentimiento informado. Luego, se incluían los datos sociodemográficos, condición migratoria, tiempo de residencia en Ecuador, conflicto y satisfacción en el vínculo con población de Quito, la escala de Anomia Subjetiva y, finalmente, respondían la Escala de Arraigo Social. El tiempo de duración varió entre 30 y 50 minutos.

En atención a estas consideraciones, la selección de la muestra se determinó como no probabilística intencional. La participación fue voluntaria y los participantes fueron identificados mediante claves numéricas, lo cual mantuvo el anonimato en el tratamiento de los datos.

\section{Resultados}

En la Tabla 1 se presentan los descriptivos de la muestra y un análisis de la comparación de los grupos. Se observaron diferencias en aspectos tales como sexo, estado civil, tiempo de estadía, nivel académico y situación laboral en Ecuador, a excepción de la edad. Las diferencias encontradas en sexo responden a la característica señalada: la población colombiana femenina es levemente mayor en tanto la cubana es principalmente masculina. A pesar de que surgieron diferencias significativas en el estado civil, ambas poblaciones presentaron una condición de casados/unión libre, seguido de solteros. Asimismo, se encontraron diferencias en el tiempo de vinculación a el Ecuador, donde los participantes cubanos presentan una migración más reciente en comparación a la muestra colombiana. En cambio, los participantes cubanos exhibieron mayor inserción laboral que los colombianos, lo que sugiere que esta última característica no se asocia al tiempo de estadía. 


\section{TABLA 1}

Descriptivos sobre características de los participantes

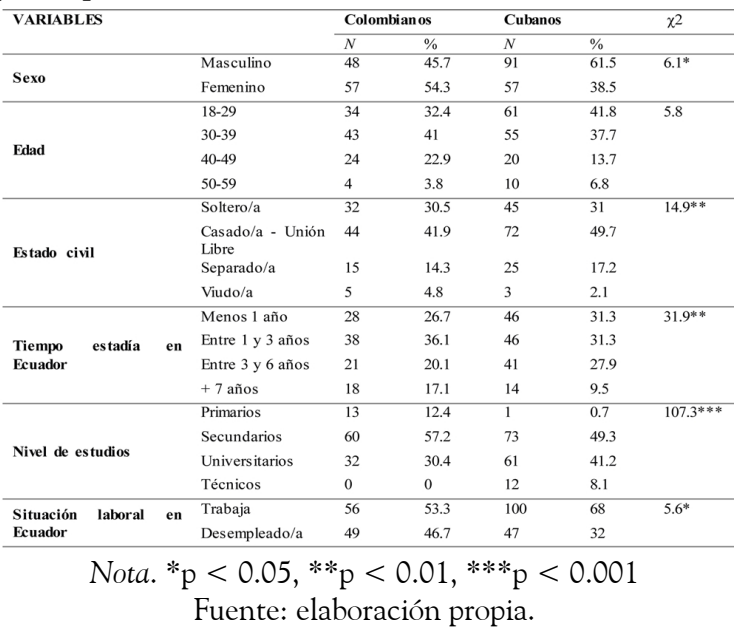

Luego se presentan las correlaciones en las distintas escalas, donde se observó una correlación directa entre Anomia y Problemas en el vínculo con la sociedad de acogida y una correlación inversa entre estas variables y casi la totalidad de las demás escalas (Tabla 2). Sin embargo, se aprecia que no se establecen relaciones significativas entre Anomia subjetiva y Vínculo satisfactorio con la población local. La variable Problemas con la sociedad de acogida solo presentó relaciones significativas con anomia subjetiva y arraigo social.

$\mathrm{Al}$ realizar comparaciones en las distintas escalas y dimensiones que las componen entre los dos grupos de participantes, se encontró lo siguiente: no hay diferencias significativas en las escalas evaluadas (todas la $p>0.05$ ), lo que revela la aparente similitud de las muestras en relación con la integración social. No obstante, las diferencias se produjeron en algunas de las dimensiones que las componen. En relación con la escala de Anomia Subjetiva, se observaron diferencias significativas en las dimensiones de exclusión social, $t(252)=-2.214, p=0.028$, siendo mayor en cubanos, $M_{\text {col }}=6.84$ y $M_{\text {cub }}=$ 7.43. En la dimensión de extrañamiento, $t(252)$ $=2.83, p=0.005$, la tendencia es contraria, $\mathrm{M}$ col $=7.48$ y $M_{\text {cub }}=6.8$. En relación con la escala de Arraigo Social, se observaron diferencias en la dimensión de arraigo cultural, $t(253)=-3.11$, $\mathrm{p}=0.002$, siendo mayor en cubanos, $\mathrm{M}$ col = 2.75 y M cub $=3.16$. Al realizar comparaciones 8 en las dimensiones de Problemas con la sociedad de acogida, se encontraron diferencias en relación

con los lugares donde ha ocurrido, $\chi^{2}=11.765$, $p=0.038$; principalmente en colombianos se produce en lugares públicos y en cubanos en el trabajo. También se observaron diferencias en las dificultades para la Adaptación al estilo de vida en Ecuador, $t(253)=2.996, p=0.008$, siendo mayores las dificultades en colombianos.

\section{TABLA 2}

Correlaciones entre las variables en estudio

\begin{tabular}{|c|c|c|c|c|c|}
\hline $\begin{array}{l}\text { VARIABLES } \\
\end{array}$ & $\begin{array}{c}\text { Anomia } \\
\text { Subjetiva }\end{array}$ & 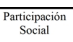 & Arraigo & $\begin{array}{l}\text { Vinculo satisfactorio } \\
\text { con sociedad acogida }\end{array}$ & $\begin{array}{l}\text { Problemas en vinculo } \\
\text { con sociedad acogida }\end{array}$ \\
\hline Anomia Subjetiva & -- & $-0.14^{*}$ & $-0.32^{* * *}$ & -0.04 & $0.2^{* *}$ \\
\hline Participación Social & -- & -. & $0.18^{* * *}$ & $0.14^{*}$ & 0.08 \\
\hline Arraigo Social & .. & .. & .- & $0.15^{* *}$ & $-0.16^{* *}$ \\
\hline $\begin{array}{l}\text { Vinculo satisfactorio } \\
\text { Con sociedad acogida }\end{array}$ & -. & -. & -- & .. & -0.04 \\
\hline $\begin{array}{l}\text { Problemas en vinculo } \\
\text { Con socicdad acogida }\end{array}$ & .- & .- & .- & .- & .. \\
\hline
\end{tabular}

Nota. $* \mathrm{p}<0.05 ; * * \mathrm{p}<0.01$

Fuente: elaboración propia.

Respecto al objetivo 1, se realizó un análisis de mediación (Hayes, 2013; Preacher \& Hayes, 2004) donde se estableció como variable dependiente la anomia subjetiva y los problemas percibidos en el vínculo con la sociedad de acogida como variable independiente. Las variables de mediación introducidas correspondieron a trabajo actual, dificultad en la adaptación al estilo de vida de Quito y el nivel de estudios. En el caso de la población colombiana, se encontró que los problemas en el vínculo se asociaron positivamente con la anomia subjetiva $(B=$ $0.42, t(105)=2.91, p=0.004)$. También se observó una relación significativa con trabajo actual $(B=-1.36, t(105)=-2.12, p=0.036)$ y adaptación al estilo de vida $(B=0.05, t(105)$ $=4.91, p=0)$. Los resultados indicaron que el mediador de adaptación al estilo de vida se asoció significativamente con anomia subjetiva $(B=3.94, t(105)=3.08, p=0.002)$. De este modo, los resultados confirmaron la mediación de las variables identificadas entre los problemas percibidos con la sociedad de acogida y anomia subjetiva $(B=0.2, S E=0.09$, $C I[0.12,0.5])$. Además, los resultados indicaron que el efecto directo de problemas percibidos con la sociedad de acogida en anomia subjetiva se convirtió en no significativa $(B=0.16, t(105)=1.04, p=0.29)$,

| Universitas Psychologica | V. i6 | No.5 | 2017 | 
cuando se controlan las variables intervinientes, lo que sugiere una mediación completa. La Figura 1a muestra los resultados.

\section{Figura la}

Mediación entre problemas en vínculo con sociedad de acogida y anomia subjetiva en participantes colombianos

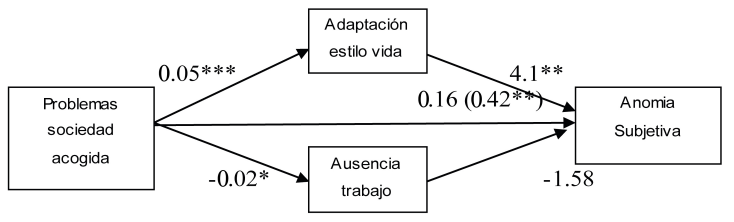

Fuente: elaboración propia.

Así, la adaptación al estilo de vida más que la ausencia de trabajo se configura como la principal variable mediadora en el caso de la población desplazada colombiana. Este resultado es llamativo, porque supondría que ecuatorianos y colombianos comparten costumbres y que este factor no estuviese relacionado con la anomia subjetiva. En otras palabras, podría esperarse que fuese la ausencia de trabajo la variable que mediara los problemas con la sociedad de acogida y la percepción de anomia, evidenciando que los patrones de la cultura ecuatoriana son percibidos como adversos en los colombianos.

En relación con la población cubana, no hubo asociación significativa entre problemas percibidos en el vínculo con la sociedad de acogida y anomia subjetiva. Se encontró una relación significativa entre problemas en el vínculo con la sociedad de acogida y trabajo actual $(B=0.02, t(142)=2.32, p=0.021) \mathrm{y}$ entre el trabajo actual con anomia subjetiva $(B=$ -2.26, $t(142)=-2.02, p=0.044)$. Los resultados confirmaron un modelo de mediación completa en este punto $(B=0.09, S E=0.05, C I[0.21$, $0.1]$ ). Los resultados se presentan en la Figura $1 \mathrm{~b}$.

\section{Figura $1 b$}

Mediación entre problemas en vínculo con sociedad de acogida y anomia subjetiva en participantes cubanos

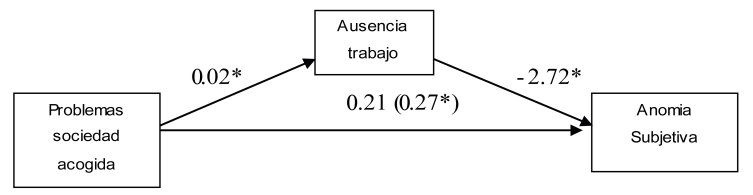

Fuente: elaboración propia.

En este caso, el modelo pone de manifiesto que en aquella población cubana que presenta problemas con la sociedad de acogida, la ausencia de trabajo es la variable que determina la percepción de anomia. Como se señaló con anterioridad, esta población es altamente calificada y quienes disponen trabajo se orientan fundamentalmente en áreas de servicios, en tanto, la población desempleada es la más expuesta a evaluar que no cumplirá sus metas sociales.

Finalmente, en relación con el objetivo 2, el análisis de mediación se realizó estableciendo al arraigo social como variable dependiente y el vínculo satisfactorio percibido con la sociedad de acogida, como independiente. Las variables intervinientes fueron conformadas por trabajo actual, mantención de costumbres culturales y origen de la migración. En el caso de la población colombiana, esta última variable se distinguió respecto a la condición migratoria en Ecuador (i.e., refugiados reconocidos o solicitantes de asilo). Primero, se encontró una relación significativa entre el vínculo satisfactorio con la sociedad de acogida y arraigo $(B=0.53, t(80)=3.76, p=0)$. El vínculo con la sociedad de acogida se asoció con la condición migratoria $(B=0.61, t(80)=2.77, p=0.007)$. Posteriormente, esta condición se relacionó con arraigo social $(B=0.17, t(80)=2.43, p=0.017)$ y trabajo actual $(B=0.45, t(80)=2.39, p=$ 0.019). Los niveles de ajuste conformaron una mediación parcial $(B=0.28, S E=0.07, C I$ $[0.04,0.35])$. Los resultados se presentan en la Figura 2a. 
Figura 2a

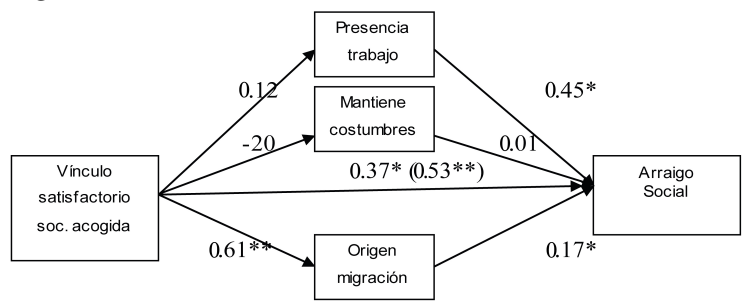

Fuente: elaboración propia.

Estos resultados sugieren que en colombianos que perciben un vínculo satisfactorio con la sociedad de acogida, la condición migratoria en que se encuentran en Ecuador es la que posibilita un mayor arraigo social. Este mayor arraigo se observa en personas refugiadas más que en otro estatus migratorio, dado que tal población ha permanecido más tiempo, dispone de mejores condiciones laborales, generalmente vive con su familia y percibe menos posibilidades de retornar a Colombia.

En la población cubana se introdujo como variable mediadora el motivo de salida de su país de origen. No se encontró relación entre el vínculo satisfactorio percibido con la sociedad de acogida y arraigo social. Se observó una asociación entre vínculo satisfactorio con el país de acogida y trabajo actual $(B=0.2, t(140)$ $=3.35, p=0.001)$. Después, se encontró una asociación entre trabajo actual y arraigo social $(B=0.31, t(140)=2.09, p=0.037)$ y con motivo de salida $(B=0.36, t(140)=2.29, p=$ $0.023)$. A pesar de estas relaciones, no se produce una confirmación del modelo de mediación $(B=$ 0.07, $\mathrm{SE}=0.06$, CI $[-0.09,0.14])$. Los resultados se muestran en la Figura $2 \mathrm{~b}$.

\section{Figura $2 b$}

Mediación entre vínculo satisfactorio con sociedad de acogida y arraigo social en participantes cubanos

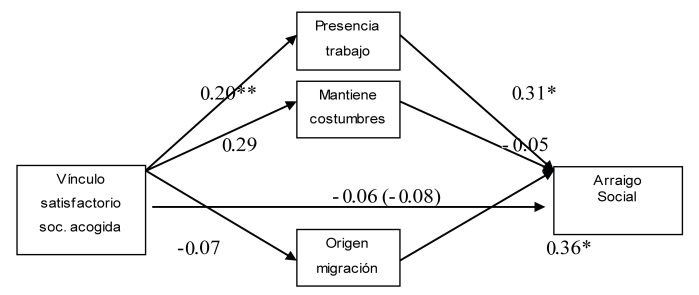

Fuente: elaboración propia.
En este caso, se observa que la presencia de trabajo tampoco configura una variable que favorezca el arraigo social de los cubanos, lo que interroga sobre qué circunstancias promoverían condiciones de integración en esta población.

\section{Discusión}

La integración social de la población en condición de movilidad constituye un desafío para las sociedades, en especial las latinoamericanas, dado el gran movimiento que se está presentando hoy en día. Las noticias que proceden desde distintos contextos informan de situaciones de exclusión y discriminación para migrantes, en el acceso a servicios sociales y puestos de trabajo que lo configuran como un grupo en alta vulnerabilidad, en especial, los que migran por condiciones de expulsión de sus países de origen. La población colombiana víctima de un conflicto armado que aún no cesa y la población cubana expuesta a condiciones de un sistema que sigue en transición política y económica y que sanciona a quienes deciden emigrar, constituyen dos de los grupos que están presentando una alta movilidad en América Latina y, por consiguiente, en Ecuador.

En este contexto, el objetivo del presente estudio es explorar las condiciones que promueven la exclusión e integración social de población migrante colombiana y cubana, en la ciudad de Quito, a través de la operativización de variables micro y macrosociales en un plano subjetivo.

Al respecto, los resultados sugieren que el vínculo percibido con la sociedad de acogida es un aspecto clave en cómo se promueve una integración o exclusión social de los grupos estudiados, en el cual tiene especial relevancia la variable de actividad laboral. En el caso de la población colombiana, cuando se presentan dificultades para acceder al trabajo y en su adaptación al estilo de vida local, conllevan percepciones anómicas de exclusión social. Es sorprendente que, dada la cercanía cultural entre Colombia y Ecuador, los participantes colombianos perciban una distancia 
en el estilo de vida respecto a la población autóctona, situación que puede relacionarse con la percepción discriminatoria que hay en Quito respecto a los colombianos. En tanto, en la población cubana únicamente la ausencia de trabajo condiciona una percepción anómica.

Por el contrario, el arraigo social está condicionado a una percepción de satisfacción en el vínculo con la sociedad de acogida, el cual aparece mediado en la población colombiana, por la presencia de actividad laboral y la condición migratoria. En este caso, resulta más significativo el estatus migratorio de refugiado reconocido que facilitaría realizar una actividad laboral. En contraste, el arraigo social en la población cubana no está asociado a alguna variable de las medidas en el presente trabajo. Aquí, cobra relevancia lo planteado respecto a que las dificultades en la integración social de grupos en movilidad humana revelan la relación que se establece con los miembros de la sociedad de acogida. Estos resultados confirman lo planteado por Correa (2013) respecto a las dinámicas relacionales que ha venido implementando la población cubana residente en Ecuador.

Los presentes resultados otorgan una señal sobre las dificultades que se presentan en los procesos de integración para personas en condición en movilidad social, en América Latina. Se podría considerar, en una lectura rápida, que ciertas similitudes en el idioma y de la riqueza cultural de las sociedades de origen, fomentarían en la población colombiana y cubana mayores posibilidades de integración social en Quito, más con una legislación constitucional ecuatoriana inédita en la región. No obstante, estos hallazgos resultan un llamado de atención a las sociedades latinoamericanas respecto a los planes y políticas de integración sobre poblaciones en condición de movilidad ya que, su ausencia o retraso en la implementación, derivan a estos grupos a varias condiciones de vulnerabilidad social. Para Ecuador, se observa una doble normativa sobre la movilidad que, a pesar de contar con un nivel de participación de ciertos colectivos, incluye una peligrosa desigualdad entre quienes son considerados migrantes. Complementario a esto, surge la necesidad de observar los imaginarios que las poblaciones autóctonas presentan de la creciente movilidad humana presente en la región, ya sea proveniente de los Estados o suscitados por los medios de comunicación. Las experiencias de exclusión social exhibidas en países del hemisferio norte que tuvieron dificultades en gestionar la llegada de población migrante, fueron resueltas en muchos casos, mediante la criminalización de los colectivos. Esta situación, aún sin ser una tendencia, está implementándose progresivamente en América Latina, lo que conlleva a crecientes dinámicas de discriminación, racismo o expulsión colectiva. De allí que resulta indispensable que los Estados implementen políticas de movilidad humana, que no se centren únicamente en aspectos de regularidad administrativa en la estadía, sino de generar condiciones sociales tanto políticas como de sensibilización en la ciudadanía, para una real integración de las personas en movilidad.

\section{Limitaciones y futuros estudios}

El presente estudio muestra algunas limitaciones que se deben considerar: en primer lugar, la elección de la muestra se realiza a partir de participantes que per se presentaban altas probabilidades de tener problemas en su IS. Esto condiciona los resultados expuestos aquí, dado que estos grupos presentan una baja vinculación $y$, en ambos grupos, hay una percepción de discriminación desde la población autóctona. Es necesario considerar en futuros estudios, grupos en condición de movilidad que provengan de culturas disímiles, que incluya poblaciones aparentemente más alejadas de dinámicas de discriminación y en la cual se podría considerar un grupo comparativo que sea representado por la propia población ecuatoriana. A pesar de esto, el aporte de la evaluación en personas de nacionalidad colombiana y cubana es que enriquece la discusión sobre la integración, la cual ha considerado a migrantes económicos, pero escasamente a quienes están presentando desplazamiento forzado. En segundo lugar, no se incorpora la dimensión objetiva de la IS, que 
ha sido enfatizada en otros estudios (Gracia et al., 1995, 2004). De este modo, la evaluación de IS planteada en este estudio no coteja la influencia de las condiciones estructurales que favorecen la anomia o arraigo social encontrados aquí. En relación con esto, no se consideraron aspectos específicos evaluados en población en condición de movilidad, tales como estatus socioeconómico, estrés sociocultural, bienestar, participación social o apoyo social. Sin embargo, este estudio consta de dos partes, donde fueron evaluados el apoyo social y la estructura de red social, que no fueron presentados en este texto.

Por último, las recientes investigaciones en países hispanoamericanos en relación con la población en condición de movilidad se han centrado en el ajuste al estrés sociocultural y sus efectos negativos y positivos (Bobowick, Basabe, \& Páez, 2015; Urzúa et al., 2015; Yáñez \& Cárdenas, 2010; Zlobina, Basabe, \& Páez, 2006). A pesar de estos hallazgos, es fundamental abordar no solo estos efectos, sino su implicancia en la relación de la población en condición de movilidad con la sociedad de acogida y en los aspectos que requieren una política de IS. Esto último es especialmente relevante para las sociedades en América Latina $y$, en este caso, Ecuador en particular, país que ha mostrado una política migratoria inclusiva en la región, dada su incorporación jurídica, pero que al mismo tiempo, es limitada por normativas de leyes orgánicas que plantean restricciones a los procesos de igualdad. Los Estados requieren promover los derechos contemplados a la población en condición de movilidad, de modo tal que contemple una educación y sensibilización a la población autóctona y no aparezca como beneficios otorgados a los extranjeros. Los resultados surgidos aquí confirman que se requieren esfuerzos en este sentido en grupos con alta vulnerabilidad.

\section{Agradecimientos}

Este trabajo fue financiado por la Pontifica Universidad Católica de Ecuador, código: K13165, denominado "Nivel de Integración
Social en inmigrantes de nacionalidad colombiana y cubana que residen en la Ciudad de Quito de febrero a noviembre de 2014". Asimismo, se agradece la colaboración de Asylum Access Ecuador, que posibilitó la puesta en contacto con población colombiana y de Ahmed Correa con la población cubana. Este trabajo está dedicado al profesor Domingo Asún Salazar (1943-2015), destacado psicólogo social y comunitario en Chile, pionero en investigaciones en integración social.

\section{Referencias}

Aceituno, R., Asún, R., Ruiz, S., Venegas, J. I., Corbalán, F., \& Reinoso, A. (2009). Anomia y alienación en estudiantes secundarios de Santiago de Chile: Resultados iniciales de un estudio comparativo 1989-2007. Psykhe, 18(2), 3-18. https://doi.org/10.4067/S0718-22282 009000200001

Alto Comisionado de las Naciones Unidas para los Refugiados. (2014). Informe Anual 2013 (Resumen ejecutivo). Quito: Autor. Recuperado de http://www.acnur.org/fileadmin/scripts/ doc.php?file $=$ fileadmin $/$ Documentos $/ \mathrm{BDL}$ /2014/9723

Alto Comisionado de las Naciones Unidas para los Refugiados. (2016). Hoja Informativa Ecuador. Quito: Autor. Recuperado de http://www.acnur.org/t3/fileadmin/Doc umentos/RefugiadosAmericas/Ecuador/20 16/ACNUR_Ecuador_2016_General_ES_ Abril.pdf

Arcentales, J. (2012). Migración cubana: recomendaciones de política pública para Ecuador incluyente. Quito: Defensoría del Pueblo.

Arcentales, J. (2017). ¿Por qué la Ley de Movilidad Humana no es una buena noticia para la igualdad de derechos? Manuscrito no publicado.

Arcentales, J., \& Chávez, G. (en prensa). La Ley Orgánica de Movilidad Humana del Ecuador: los límites de la regularidad 
e irregularidad de la movilidad. En N. Caicedo (Coord.), Tendencias y retos de las políticas y reformas migratorias en América Latina. Un estudio comparado. Barcelona.

Asún, D., Alfaro, J., \& Morales, G. (1994). Análisis crítico del uso de categorías y estrategias para el estudio e intervención psicosocial con jóvenes en Chile. Revista Chilena de Psicología, 15 (1), 5-14.

Asún, D., Alfaro, J., Fernández, P., Báez, X., Pérez, L., \& Vergara, R. (1998). Construcción de un modelo teórico-metodológico para la medición de la integración social (Proyecto FONDECYT № 1980190). Santiago: FONDECYT.

Bobowick, M., Basabe, N., \& Páez, D. (2015). The bright side of migration: Hedonic, psychological, and social wellbeing in immigrants en Spain. Social Science Research, 51, 189-204. https://doi.org/10.10 16/j.ssresearch.2014.09.011

Castel, R. (1997). Las metamorfosis de la cuestión social. Una crónica del salariado. Buenos Aires: Paidós.

Cohen, S., Gottlieb, B., \& Underwood, L. (2000). Social relationships and health.

Correa, A. (2013). Inserción laboral y producción de espacios: la migración cubana en Ecuador (Experiencias latinoamericanas). Questiones Urbano Regionales, 1(3), 39-64. Recuperado de http://repositorio.flacsoandes.edu.ec/bit stream/10469/6461/3/REXTN-QUR3-03Correa.pdf

Cowen, E. (2000). Community psychology and routes to psychological wellness. En J. Rappaport \& E. Seidman (Eds.), Handbook of community psychology (pp. 79-99). Nueva York: Kluwer/Plenum.

Cuellar, I., Bastida, E., \& Braccio, S. M. (2004). Residency in the United States, subjective well-being, and depression in an older Mexican-origin sample. Journal of Aging and Health, 16, 447-466. https://doi.org/10.117 7/0898264304265764

De Luca, S., Bobowick, M., \& Basabe, N. (2011). Adaptación sociocultural de inmigrantes brasileños en el
País Vasco: bienestar y aculturación. Revista de Psicología Social, 26(2), 275-294. http://www.academia.edu/19885 17/Adaptacion_sociocultural_de_inmigran tes..._Basque_Country

Durkheim, E. (1989). El suicidio. Madrid: Akal. República del Ecuador, Ley Orgánica de Movilidad Humana (2017). [Asamblea Nacional. En Pleno]. Por la cual se regula el ejercicio de derechos, obligaciones, institucionalidad y mecanismos vinculados a las personas en movilidad humana, que comprende emigrantes, inmigrantes, personas en tránsito, personas ecuatorianas retornadas, quienes requieran de protección internacional, víctimas de los delitos de trata de personas y de tráfico ilícito de migrantes; y, sus familiares. Febrero 6 de 2017. Registro Oficial No. 938.

Gracia, E., \& Herrero, J. (2004). Determinants of social integration in the community: An exploratory analysis of personal, interpersonal and situational variables. Journal of Community $\mathcal{E}$ Applied Social Psychology, 14, 1-15. https://doi.org/10.100 2/casp.746

Gracia, E., García, F., \& Musitu, G. (1995). Macrosocial determinants of social integration: Social class and area effect. Journal of Community E Applied Social Psychology, 5, 105-119. Recuperado de https ://www.uv.es/ egracia/enriquegracia/docs/ scanner/Macrosocial\%20Determinants $\% 2$ 0JCASP\%201995.pdf

Hayes, A. F. (2013). Introduction to mediation, moderation, and conditional process analysis: A regression-based approach. Nueva York: Guilford Press.

Herrera, G. (2008). Ecuador: la migración internacional en cifras. Quito: UNFPA y Flacso Ecuador.

Herrero, J., \& Gracia, E. (2007). Measuring perceived community support: Factorial structure, longitudinal invariance and predictive validity of the PCSQ (Perceived Community Support Questionnaire). Journal of Community Psychology, 35, 
197-217. https://doi.org/10.1002/jcop.2014 3

Herrero, J., Gracia, E., Fuente, A., \& Lila, M. (2012). Desorden social, integración social y bienestar subjetivo en inmigrantes latinoamericanos en España. Anales de Psicología, 28(2), 505-514. https://doi.org/1 0.6018/analesps.28.2.148721

House, J. S., Umberson, D., \& Landis, K. R. (1988). Structures and processes of social support. American Review of Sociology, 14, 293-318. https://doi.org/10.1146/annurev.s o.14.080188.001453

Jaramillo, A. M. (2008). Contextos explicativos del desplazamiento interno y del refugio de colombianos en Ecuador y Canadá. En P. Riaño \& M. Villa (Eds.), Poniendo tierra de por medio. Migración forzada de colombianos en Colombia, Ecuador y Canadá (pp. 37-69). Medellín: Corporación Región.

Laparra, M., Obradors, A., Pérez, B., Pérez, M., Renes, V., Sarasa, S.,... Trujillo, M. (2007). Una propuesta de consenso sobre el concepto de exclusión. Implicaciones metodológicas. Revista Española del Tercer Sector, 5, 15-57. Recuperado de https://dialnet.unirioja.es/se rvlet/articulo?codigo $=2376685$

Preacher, K. J., \& Hayes, A. F. (2004). SPSS and SAS procedures for estimating indirect effects in simple mediation models. Behavior Research Methods, Instruments, $\mathcal{E}$ Computers, 36(4), 717-731. Recuperado de http://quantpsy.org/pubs/preacher_haye s_2004.pdf

Sozanski, A., Sarmiento, K., \& Reyes, C. (2016). Derecho al trabajo en personas refugiadas y solicitantes de asilo en Ecuador: dificultades y propuestas. Forced Migration Review, 51, 93-95. Recuperado de http://www.fmreview.org/sites/fmr/files/ FMRdownloads/en/destination-europe/soz anski-sarmiento-reyes.pdf

Torrente, G., Ruiz-Hernández, J. A., Ramírez, M. C., \& Rodríguez, Á. (2011).Construcción de una escala para medir el arraigo en inmigrantes latinoamericanos. Anales de Psicología, 27(3), 843-851. Recuperado de http://revistas.um.es/analesps/article/vie w/135591

Urzúa, A., Vega, M., Jara, A., Trujillo, S., Muñoz, R., \& Caqueo-Urízar, A. (2015). Calidad de vida percibida en inmigrantes sudamericanos en el norte de Chile. Terapia Psicológica, 33(2), 139-156. Recuperado de http://www.scielo.cl/pdf/terpsicol/v33n2 /art08.pdf

Valdenegro, B. A. (2005). Factores psicosociales asociados a la delincuencia juvenil. Psykhe, 14(2), 33-42. https://doi.org/10.4067/S071 $8-22282005000200003$

Ward, C., \& Kennedy, A. (1999). The measurement of sociocultural adaptation. International Journal of Intercultural Relations, 23(4), 659-678. Recuperado de http://isplaza.dreamhosters.com/wp-con tent/uploads/2014/05/SCAS-Ward-Kenne dy-1999.pdf

Yáñez, S., \& Cárdenas, M. (2010). Estrategias de aculturación, indicadores de salud mental y bienestar psicológico en un grupo de inmigrantes sudamericanos en Chile. Salud y Sociedad, 1(1), 51-70. Recuperado de http://www.redalyc.org/pdf/4397/43974 2462006.pdf

Zarza, M. J., \& Sobrino, M. I. (2007). Estrés de adaptación sociocultural en inmigrantes latinoamericanos residentes en Estados Unidos vs. España: una revisión bibliográfica. Anales de Psicología, 23(1), 72-84. Recuperado de http://www.um.es/an alesps/v23/v23_1/10-23_1.pdf

Zlobina, A., Basabe, N., Páez, D., \& Furnham, A. (2006). Sociocultural adjustment of immigrants: Universal and groupspecific predictors. International Journal of Intercultural Relations, 30, 195-211. Recuperado de https://scholar.google.es/cit ations? user $=s$ Wevq7YAAAAJ

\section{Notas}

* Artículo de investigación 